\title{
JEJUNOGASTRIC INTUSSUSCEPTION: AN UNUSUAL CAUSE OF HAEMATEMESIS
}

\author{
MOHIBUR RAHMAN ${ }^{1}$, AKRAM HOSSAIN ${ }^{2}$, ROBED AMIN ${ }^{3}$, NASIR UDDIN ${ }^{4}$, TANVIR HOSSAIN ${ }^{5}$, \\ ANJAN KUMAR SAHA ${ }^{5}$, ARIFUR RAHMAN ${ }^{5}$, TANVIR HOSSAIN ${ }^{6}$
}

\begin{abstract}
Intussusception most commonly occurs in children and is rare in adults, accounting for only $5 \%$ of all cases of intussusceptions. Intussusception following gastric surgery is an extremely rare postoperative complication. We present a case where following gastrojejunostomy with truncal vagotomy a retrograde intussusception of the efferent limb into the gastrojejunostomy stoma occurred. The intussusception was well visualized by upper GI endoscopy prior to surgery. Exploratory laparotomy was done within 24 hours after the onset of the symptoms. After resection of loop of intussusceptum, gastrojejunostomy and jejunoileostomy was done.
\end{abstract}

Keyword: Intususception, Intestinal invagination, Jejunogastric intussusception

\section{Introduction}

Intussusception most commonly occurs in childhood and is rare in adults, accounting for only $5 \%$ of all cases of intussusceptions. ${ }^{1}$ Intussusception following gastric surgery is an extremely rare postoperative complication. ${ }^{2}$ It may develop in clinical situations following gastroenterostomy, Billroth II gastric surgery with or without Braun anastomosis, or Rouxen-Y gastrojejunostomy. ${ }^{2}$ Jejunogastric intussusception (JGI) is a rare complication of gastrectomy or gastrojejunostomy. ${ }^{3}$

It can occur from days to years after the procedure. Its estimated frequency is approximately $0.1 \%$ and close to 300 cases had been recorded in the medical literature. ${ }^{4}$ Intussusception has two clinical patterns - an acute type and a chronic course with episodes of recurrence. In acute form, haematemesis can be seen. ${ }^{5}$ The mortality may be up to $50 \%$ in these cases, especially if corrective operation is delayed for more than 48 hours. ${ }^{2,3}$ Little is known about the mechanism underlying the condition. ${ }^{2}$ Preoperative diagnosis can be made by barium meal or endoscopic examination. But pre-operative endoscopic confirmation has been reported infrequently. ${ }^{5}$ Surgical reduction with laparotomy is mandatory, although definitive corrective and preventative measures have not yet been established. ${ }^{2}$ In present case, following gastrojejunostomy with truncal vagotomy a retrograde intussusception of the efferent limb into the gastrojejunostomy stoma occurred. The intussusception was well visualized by upper GI endoscopy prior to surgery.

\section{Case report}

The patient was a 56 years old male who was admitted with severe upper abdominal pain for 12 hours, repeated vomiting of altered blood for 10 hours and lumpy feeling in epigastrium. He had no history of melaena. He also gave history of periodic epigastric pain for one year and epigastric fullness for 6 month. He had no history of jaundice, or taking NSAIDS. He gave history of gastrojejunostomy with truncal vagotomy for duodenal ulcer perforation 19 years back.

On examinations-he was found ill looking with moderate anaemia, not icteric, his pulse rate was 102 beats/min, blood pressure was-100/60 $\mathrm{mm}$ of $\mathrm{Hg}$,

1. Associate Professor, Department of Gastroenterology, Uttara Adhunik Medical College, Dhaka.

2. Associate Professor, Department of Surgery, Uttara Adhunik Medical College, Dhaka

3. Assistant professor, Department of Medicine, Dhaka Medical College, Dhaka

4. Assisatant Registrar, Department of Gastroenterology, Uttara Adhunik Medical College, Dhaka.

5. Medical officer, Department of Gastroenterology, Uttara Adhunik Medical College, Dhaka.

6. Assistant Registrar, Department of Surgery, Uttara Adhunik Medical College, Dhaka

Correspondence: Dr Mohammad Mohibur Rahman, Associate Professor, Department of Gastroenterology, Uttara Adhunik Medical College, Plot-34, Road-4, Sector-9, Uttara, Dhaka-1230. E-mail: rmohammadmohibur@yahoo.com 
temperature- $99^{\circ} \mathrm{F}$, respiratory rate- 16 breaths $/ \mathrm{min}$. On per abdominal examination- there was a visible swelling in the upper abdomen. The lump was firm, irregular surface and tender and was situated in the epigastrium and left upper quadrant. The lump moved with respiration, but there was no visible peristalsis or bruit.

Other systemic examinations were normal. On investigations, haemoglobin was- $11.8 \mathrm{gm} / \mathrm{dl}$, Total count of WBC- 9X109/1. Polymorph- 65\%, Lymphocytes- 30\%, Platelets-260x10 $/$ l. ESR- $24 \mathrm{~mm}$ in 1st hour. Ultrasonogram of whole abdomenexcessive bowel gas in the upper abdomen and no other abnormality. Endoscopy of upper GIT- showed large amount of altered blood in the stomach. Prolapsed, lobulated and engorged loops of jejunum seen in the stomach with oozing of blood from the loops that occupied the entire body of the stomach. The bulb of the duodenum and post bulbar area was normal. We diagnosed the case as jejunogastric intussusception with necrosis of jejunum.

After resuscitation with intravenous fluid and electrolytes emergency exploratory laparotomy was done within 24 hours after the onset of the symptoms. After opening of the peritoneal cavity jejunum was found inside the stomach. The stomach was hugely distended containing long segments of gangrenous jejunum. The loop was resected from the pyloric end of the stomach to distal jejunum followed by closure of duodenum stump, gastrojejunostomy and jejunoileostomy was done (Figure 1). The post operative period was uneventful. After being discharge he was follow up in the out -patient department for one month and he remained quite well.

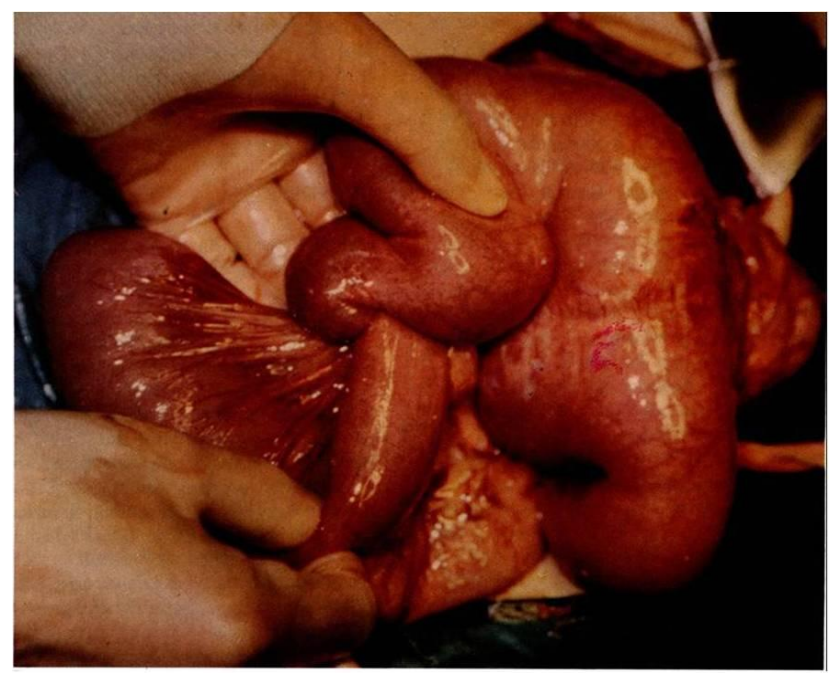

Fig-1: Intussusception of jejunum efferent loop

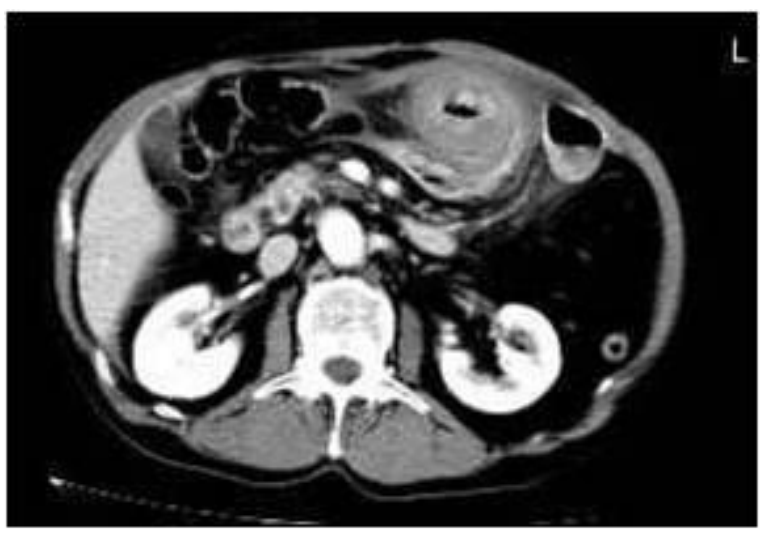

Fig-2: Target appearance of intussusception in $C T$ scan.

\section{Discussion}

Jejunogastric intussusception was first described by Bozzi in 1914, thirty years after the first gastrojejunostomy was performed. ${ }^{5}$ Intussusception of the jejunum into the stomach via a gastrointestinal stoma, a rare complication of gastric surgery and it is known as jejunogastric intussusception (JGI). ${ }^{5,6}$ Three anatomic types of JGI have been described: type I concerns the afferent loop, type II the efferent loop and type III represents a combined form. It has been stated that type II or retrograde efferent loop intussusception is the most common (80\%) with the two other types accounting for $10 \%$ each. In the case presented, a type II JGI was documented. ${ }^{3,7}$

Another means of classification noted in the literature is by clinical course, acute or chronic. ${ }^{5}$ The acute type presents with classical triads - sudden onset of epigastric pain, vomiting of food, then bile and later blood and palpable movable epigastric mass. Hametemsis implies complete obstruction and bleeding originates from the necrotic jejuna mucosa. The blood is usually dark red and rarely bright red. A palpable, tender mass is noted in approximately one half of the patients. The acute type occurs usually within the first day postoperatively but may occur after a year. In our case the time elapsed after the operation was 16 years. Chronic type usually has later onset. ${ }^{5}$ In the chronic form, the symptoms may be roughly similar to the acute form but milder, transient and subside spontaneously. Thus, the patient complains for recurrent episodes of upper abdominal discomfort that is exacerbated by the food and usually subsides 1-1.5 h after the meal. Nausea and vomiting can also be present but epigastric pain is the main complain of patient. ${ }^{3,7}$ 
The pathogenesis is unknown, but several possible causes had been discussed e.g. functional causes like antiperistalsis triggered possible $\mathrm{e}^{3,5}$ possibly by hyperacidity or spasm, mechanical causes like increased intra-abdominal pressure, adhesions developing after laparotomy, derangements in stomal function produced by vomiting, long afferent loop, jejunal spasm with abnormal motility, retrograde peristalsis etc. ${ }^{3-5}$ Probably, retrograde peristalsis, which can occur in normal people prior to gastric surgery, seems to be accepted as the cause of type II JGI by most authors. ${ }^{3}$

Early diagnosis is of paramount importance. ${ }^{5}$ Endoscopy remains the main diagnostic tool and an alternative to surgical treatment. However, because the condition is so rare and is seldom suspected in the initial presentation, other diagnostic procedurs are frequently done before endoscopy. The endoscopic findings of intussusception have been described infrequently. Large reddish blue intestinal loops filling within the lumen of stomach or enteroanastomotic loop with or without dark red blood can be seen. ${ }^{5}$ Plain X ray of abdomen may reveal a homogenous mass outlined by intragastric air.

In barium meal typical coil spring appearance within the gastric pouch or enteroanastomotic loop can be demonstrated. ${ }^{5}$ In USG, typical findings are an echogenic centre surrounded by concentric echogenic rings with a peripheral rim of hypoechogenicity. ${ }^{4}$ Computed tomography is gaining wide acceptance as a diagnostic tool in abdominal pain and acute abdomen. In jejunogastric intussusceptions, computed tomography findings are characteristic and similar to enteroenteric intussusception. A target appearance (Figure 2), with a low-attenuation component that is crescent shaped, swirl-like and eccentric, representing mesenteric fat, are the common findings. The condition progresses to ischemia through 4 stages: initially a target mass is seen (stage 1), followed by a layering pattern (stage 2 ), loss of the layering pattern due to edema (stage 3) and, finally, bowel thickening and loss of fascial planes (stage 4). The presence of intramural air can also indicate bowel necrosis. ${ }^{4}$

In the chronic form, the diagnosis is difficult. In many of such patients, the correct diagnosis has never been established. The main reason for this is that upper GI series or upper GI endoscopy must be performed during the symptomatic period for the diagnosis to be confirmed. However, it has been suggested that in the asymptomatic period, the provocation of JGI during endoscopy by the use of a jet of water directed towards the anastomotic stoma may be diagnostic of the chronic form. ${ }^{3}$

Even though jejunogastric intussusception is a rare complication after gastric surgery, early diagnosis is important, because immediate treatment is warranted in the acute type. Most authors report a mortality of $10 \%$ if operation is performed within 48 hours after the onset of severe symptoms and as high as $50 \%$, if operation is delayed. ${ }^{5}$

In our case operation was performed within 24 hours after the onset of symptoms.

Treatment of acute type is always surgical, with reduction of intussusceptum, if viable and reducible, or resection of the bowel, if it is necrotic. ${ }^{5}$

Some author have described a desinvagination either by the endoscopy or by the weight of the contrast material at the time of upper GI series, in chronic type. 5

Surgical options include reduction, resection, revision of the anastomosis and the take-down of the anastomosis, depending on the conditions found during the operation. The best way to prevent recurrence, if any, has not been identified yet. ${ }^{3}$ To prevent recurrence the reduced jejunum should be fixed either to the afferent limb of gastrojejunal anastomosis or to the transverse mesocolon. ${ }^{7}$ The treatment of chronic recurrent variety of jejunogastric intussusception is symptomatic. If symptoms persist then revisional surgery is performed. 7

In conclusion, JGI should be considered in all patients with a history of gastrectomy who present with colicky abdominal pain, vomiting and hematemesis. Emergency endoscopy is quite valuable in the diagnosis.

\section{References}

1. Senol A, $I^{\circ}$ ler M, Songür Y, et al. Jejunogastric intussusception: a rare cause of hematemesis. Turk J Gastroenterol 2009; 20: 74-5.

2. Kim KH, Jang MK, Kim HS, et al. Intussusception after Gastric Surgery. Endoscopy 2005; 37: 12371243. 
3. Archimandritis AJ, Hatzopoulos N, Hatzinikolaou $\mathrm{N}$, et al. Jejunogastric intussusception presented with hematemesis: a case presentation and review of the literature. Gastroenterol 2001; 1: 1.

4. Herbella FAM, Del Grande JC. Radiology for the surgeon Soft-tissue case 53. Can J Surg 2003; 46: 465-466.

5. Jang WI, Kim ND, Bae SW, et al. Intussusception into the enteroanastomosis after Billroth II gastric resection; diagnosed by gastroscopy. J Korean Med Sci 1989; 4: 51-54.

6. Goverman J, Greenwald M, Gellman L, Gadaleta D. Antiperistaltic (retrograde) intussusception after Roux-en-Y gastric bypass. Am Surg 2004; 70: 67-70.

7. Gupta SS, Singh GG. Retrograde jejunogastric intussusception: an unusual cause of haematemesis (a case report). J Postgrad Med 1986; 32:105. 